\title{
ADSORÇÃO DE Cr(VI) EM ESFERAS RETICULADAS DE QUITOSANA - NOVAS CORRELAÇÕES CINÉTICAS E TERMODINÂMICAS UTILIZANDO MICROCALORIMETRIA ISOTÉRMICA CONTÍNUA
}

\author{
Rivaldo Cardoso Silva, Marcos Antonio S. Andrade Jr. e Antonio Reinaldo Cestari* \\ Departamento de Química, Centro de Ciências e Estudos de Tecnologia, Universidade Federal de Sergipe, 49100-000 São Cristóvão \\ - SE, Brasil
}

Recebido em 3/7/09; aceito em 22/10/09; publicado na web em 2/3/10

\begin{abstract}
ADSORPTION OF Cr(VI) ONTO CROSSLINKED CHITOSAN MICROBEADS - NEW KINETIC AND THERMODYNAMIC CORRELATIONS USING CONTINUOUS ISOTHERMAL MICROCALORIMETRY. The synthesis and characterization of crosslinked chitosan microbeads and their application in the removal of $\mathrm{Cr}(\mathrm{VI})$ are described. New kinetic and thermodynamic parameters of $\mathrm{Cr}(\mathrm{VI})$ adsorptions processes were found using continuous isothermal calorimetry. All adsorption processes are exothermic in nature. However, a multivariate statistical analysis have pointed out that adsorption enthalpies were affected by important binary interactions of the initial $\mathrm{Cr}(\mathrm{VI})$ in solution and temperature. The adsorption energetic data were well fitted to a kinetic exponential model, which have indicated fractionary adsorption kinetic orders.
\end{abstract}

Keywords: chitosan; $\mathrm{Cr}(\mathrm{VI})$ adsorption; microcalorimetry.

\section{INTRODUÇÃO}

A quitina é um polissacarídeo biodegradável e de baixa toxidez, largamente encontrada em invertebrados marinhos, terrestres e em fungos, sendo o segundo biopolímero mais abundante presente na natureza, depois da celulose. ${ }^{1-3}$ A quitosana é o produto da reação, em meio alcalino, da desacetilação da quitina. ${ }^{4} \mathrm{~A}$ principal diferença entre ambas encontra-se no carbono- 2 dos anéis glicosídicos de suas estruturas. Na quitina, há a presença de grupos acetil $\left(-\mathrm{CO}-\mathrm{CH}_{3}\right)$ e na

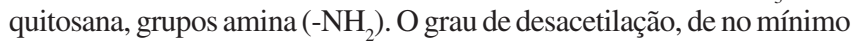
$65 \%$, induz e controla a maior parte das propriedades das quitosanas. ${ }^{5}$

As reações de reticulações cruzadas conferem à quitosana uma excelente estabilidade química e mecânica, mesmo em meio ácido. ${ }^{6}$ Um dos principais compostos que promovem esse tipo de reação na quitosana é a epicloridrina, que apresenta a vantagem de reagir, preferencialmente, pelos grupos hidroxila do carbono-6 da cadeia polimérica. Assim, os grupos aminados do carbono-2 ficam mais disponíveis para interações químicas específicas. ${ }^{7}$

Quando os grupos amina se encontram protonados $\left(-\mathrm{NH}_{3}^{+}\right)$, a quitosana pode interagir com espécies aniônicas em solução, como dextranssulfato de sódio, ácido salicílico, surfactantes aniônicos e corantes aniônicos, tipicamente por meio de interações eletrostáticas, ligações de hidrogênio e interações hidrofóbicas. Vários tipos de metais tóxicos, como o cromo, também formam espécies aniônicas em solução, ${ }^{8}$ apresentando, portanto, uma grande afinidade pela quitosana.

O cromo é um metal pesado encontrado em rochas, animais, plantas e solos, podendo formar uma grande variedade de compostos com estados de oxidação entre 0 e +6 . $O$ cromo trivalente é um elemento traço essencial para os humanos. Contudo, os compostos de cromo (VI), por serem fortemente oxidantes, são muito nocivos à saúde. Tais compostos se apresentam em solução aquosa, principalmente, nas formas de $\left[\mathrm{CrO}_{4}\right]^{2-},\left[\mathrm{Cr}_{2} \mathrm{O}_{7}\right]^{2-}, \mathrm{HCrO}_{4} \mathrm{e} \mathrm{H}_{2} \mathrm{CrO}_{4}{ }^{9}$ Estas espécies podem penetrar nas membranas biológicas e causar danos às estruturas celulares. A intoxicação torna-se séria quando há adsorção pelo trato intestinal. Assim, torna-se necessário limitar a descarga de $\mathrm{Cr}(\mathrm{VI})$ ou removê-lo de efluentes aquáticos. ${ }^{10}$

\footnotetext{
*e-mail: cestari@ufs.br
}

Há diversos processos para realizar o tratamento de efluentes contendo metais pesados, dentre os quais a precipitação cáustica, eletrólise, evaporação e osmose reversa. Contudo, estes métodos apresentam vários inconvenientes, como alto consumo de energia, aplicabilidade limitada e baixa capacidade de remoção. ${ }^{11} \mathrm{O}$ fenômeno da adsorção, principalmente envolvendo interfaces sólido/solução, tem se mostrado adequado para a remoção de $\mathrm{Cr}(\mathrm{VI})$ de soluções aquosas diluídas.

Dado o grande potencial de aplicação da adsorção, um conhecimento prévio dos parâmetros cinéticos e termodinâmicos inerentes aos processos de interação que ocorrem na interface sólido/solução é extremamente necessário, pois propiciam uma boa fundamentação para se prever o comportamento dos materiais adsorventes nas mais diferentes situações experimentais. ${ }^{12}$ Vários parâmetros importantes sofrem variações em função do tempo de contato do adsorvente na solução, da concentração inicial do adsorvato em solução, da temperatura de adsorção, dentre outros fatores experimentais.

Neste trabalho foi estudada a interação de Cr(VI) em solução aquosa com esferas de quitosana reticuladas. Os experimentos foram conduzidos em uma célula calorimétrica acoplada a um calorímetro Tian-Calvet, operando em modo isotérmico. Os resultados obtidos permitiram estabelecer novas correlações entre dados cinéticos, termodinâmicos e as quantidades adsorvidas de $\mathrm{Cr}(\mathrm{VI})$ nas esferas de quitosana.

\section{PARTE EXPERIMENTAL}

\section{Materiais e reagentes}

A quitosana com grau de desacetilação de $75 \%$ foi cedida pela Empresa C. E. Roeper, da Alemanha. Sua caracterização encontra-se descrita em estudos anteriores. ${ }^{13}$ Os reagentes de pureza analítica: epicloridrina, hidróxido de sódio, ácido acético e dicromato de potássio foram obtidos da Synth e utilizados sem purificação prévia.

\section{Preparação das esferas de quitosana}

Para o preparo das esferas de quitosana, ${ }^{13} 15,0 \mathrm{~g}$ de quitosana em pó foram completamente dissolvidos em $500 \mathrm{~cm}^{3}$ de uma solução 
de ácido acético $0,140 \mathrm{~mol} \mathrm{dm}^{-3}$ sob constante agitação, durante 24 h. O gel formado foi gotejado em uma solução constituída de 50,0 g de $\mathrm{NaOH}$ e $100,0 \mathrm{~cm}^{3}$ de etanol a $95 \%$, utilizando-se uma bureta de $50 \mathrm{~cm}^{3}$, sob agitação magnética constante. As esferas formadas foram lavadas abundantemente com água desionizada e etanol. Em seguida, foram secas em estufa por $2 \mathrm{~h}$ a $330 \mathrm{~K}$.

\section{Reticulação das esferas de quitosana}

Cerca de $15,0 \mathrm{~g}$ de esferas de quitosana foram adicionados a $100 \mathrm{~cm}^{3}$ de água bidestilada. Em seguida, adicionou-se uma solução de $\mathrm{NaOH} 0,01$ mol dm${ }^{-3}$, até a solução apresentar $\mathrm{pH} 10$. Foram adicionados $5,7 \mathrm{~cm}^{3} \mathrm{de}$ epicloridrina, sendo a suspensão agitada mecanicamente durante $2,0 \mathrm{~h}$ a $313 \mathrm{~K}$. As esferas reticuladas foram lavadas abundantemente com água desionizada e etanol. Finalmente, foram secas em estufa a $330 \mathrm{~K}$, durante 2 h. $^{13}$

\section{Caracterização das esferas por FTIR}

Os espectros de absorção na região do infravermelho das esferas reticuladas e não reticuladas foram obtidos na faixa de $4000 \mathrm{a} 400 \mathrm{~cm}^{-1}$, com uma resolução de $4 \mathrm{~cm}^{-1}$, utilizando-se um espectrofotômetro modelo Spectrum BX, com transformada de Fourier (FTIR), da Perkin Elmer. As esferas foram pulverizadas em um almofariz juntamente com $\mathrm{KBr}$ e transformadas em pastilhas finas por prensagem.

\section{Estudos calorimétricos da adsorção de $\mathrm{Cr}$ (VI)}

Os experimentos de adsorção foram realizados em uma célula calorimétrica de aço inox acoplada a um calorímetro do tipo TianCalvet, modelo C80, da Setaram Instruments, utilizando-se o sistema de quebra de membrana. ${ }^{14}$ De maneira sucinta, em um experimento típico, uma massa de aproximadamente $100 \mathrm{mg}$ das esferas foi colocada na parte inferior da célula calorimétrica. Na parte superior, adicionaram-se $3,0 \mathrm{~cm}^{3}$ de solução de dicromato de potássio, preparada em tampão de HAc/NaAc pH 4,0. Uma membrana de Teflon ${ }^{\circledR}$ fina separou as soluções, sendo toda a célula estabilizada em uma temperatura pré-estabelecida. Quando se observou a formação de uma linha-base estável (precisão da ordem de $\pm 10^{-4} \mathrm{~mW}$ ), em um gráfico de potência $(\mathrm{mW})$ vs tempo (min), a membrana foi rompida com auxílio de uma haste móvel, sendo o processo de adsorção acompanhado de maneira contínua até o retorno da linha-base à sua posição original. A célula calorimétrica foi imediatamente aberta e uma alíquota da solução de $\mathrm{Cr}(\mathrm{VI})$ foi retirada para a determinação da concentração de equilíbrio. Os experimentos foram realizados em duplicata utilizando-se soluções de $\mathrm{Cr}(\mathrm{VI})$ nas concentrações de $5,0 \times 10^{-4}$ e $5,0 \times 10^{-3} \mathrm{~mol} \mathrm{dm}^{-3}$, nas temperaturas de 308 e $318 \mathrm{~K}$. As energias dos processos de adsorção de $\mathrm{Cr}(\mathrm{VI}), Q_{a d s}\left(\mathrm{~J} \mathrm{~g}^{-1}\right)$ e os tempos totais dos processos $\left(T_{r}\right)$ foram calculados por integrações das curvas calorimétricas, utilizando-se o software Setsoft, da Setaram Instruments.

As interações dos componentes do solvente tamponado com as esferas de quitosana também são detectadas em experimentos conduzidos por calorimetria isotérmica. Para subtrair o efeito da interação, $\mathrm{Q}_{\mathrm{m}}$, realizaram-se testes calorimétricos de "brancos", substituindo-se a solução de $\mathrm{Cr}(\mathrm{VI})$ pelo solvente tamponado. Assim, as energias resultantes relacionadas às interações esferas de quitosana/Cr(VI), $Q_{i n t}$, foram calculadas através da Equação 1: ${ }^{15}$

$Q_{\text {int }}=Q_{a d s}-Q_{m}$

As determinações quantitativas de $\mathrm{Cr}(\mathrm{VI})$, após cada registro dos efeitos térmicos das energias de adsorção, $Q_{a d s}$, foram realizadas pelo método espectrofotométrico da difenilcarbazida (limite de detecção em torno de $\left.0,5 \mathrm{mg} \mathrm{dm}^{-3}\right) .{ }^{16} \mathrm{~A}$ quantidade de $\mathrm{Cr}(\mathrm{VI})$ adsorvida $(N)$ em cada experimento calorimétrico de adsorção foi calculada utilizandose a Equação 2:17

$N=\frac{\left(C_{i}-C_{f}\right) V}{m}$

onde $N$ é a quantidade de $\mathrm{Cr}(\mathrm{VI})$ adsorvido em ( $\left.\mathrm{mol} \mathrm{g}^{-1}\right) ; C_{i}$ e $C_{f}$ são as concentrações inicial e final de cromo em solução (em mol dm${ }^{-3}$ ), respectivamente; $V$ é o volume da solução de $\mathrm{Cr}(\mathrm{VI})$ em $\mathrm{dm}^{3}$ e $m$ é a quantidade (em g) de esferas presentes na célula calorimétrica.

\section{RESULTADOS E DISCUSSÃO}

Na Figura 1 é apresentado um esquema de formação da quitosana reticulada com epicloridrina. A preferência pelo uso da quitosana na forma de esferas deveu-se ao fato destas apresentarem maiores estabilidades mecânicas e facilidades operacionais em relação à quitosana em pó. ${ }^{18}$ Em um estudo anterior, foram determinadas as densidades das esferas, antes e após a reticulação com epicloridrina. ${ }^{13}$ Elas apresentaram aproximadamente $1,0 \mathrm{~mm}$ de diâmetro e densidades de 7,13 $\pm 0,34 \times 10^{-4}$ e $4,19 \pm 0,22 \times 10^{-4} \mathrm{~g} / \mathrm{mm}^{3}$, antes e após a reticulação, respectivamente. A diminuição das densidades das esferas ocorreu devido aos rearranjos da estrutura interna da quitosana, proporcionado pela inclusão das moléculas de epicloridrina. ${ }^{13}$
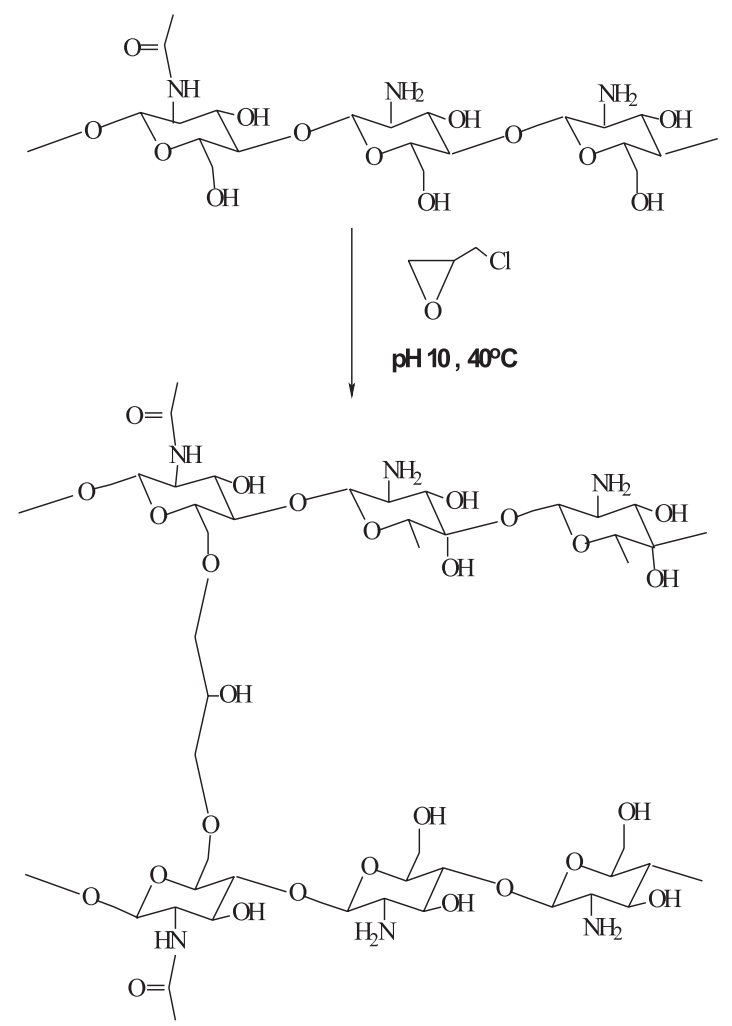

Figura 1. Esquema da reação das esferas de quitosana com a epicloridrina. Detalhes experimentais: temperatura $40{ }^{\circ} \mathrm{C}, \mathrm{pH} 10,0$, agitação constante durante $2 h$

\section{Caracterização por FTIR}

Os espectros de FTIR das esferas não reticuladas e reticuladas com epicloridrina são mostrados na Figura 2. Comparando-se os espectros, é possível observar que a banda larga em torno de 3400 
$\mathrm{cm}^{-1}$, atribuída aos estiramentos dos grupos hidroxila (-OH) do polissacarídeo, diminuiu de intensidade, sugerindo a ocorrência da reação preferencial de reticulação pelos grupos $\mathrm{OH}$ da quitosana. ${ }^{13}$ Vários trabalhos da literatura também relatam esse tipo de reação preferencial da epicloridrina com a quitosana. ${ }^{3,4}$

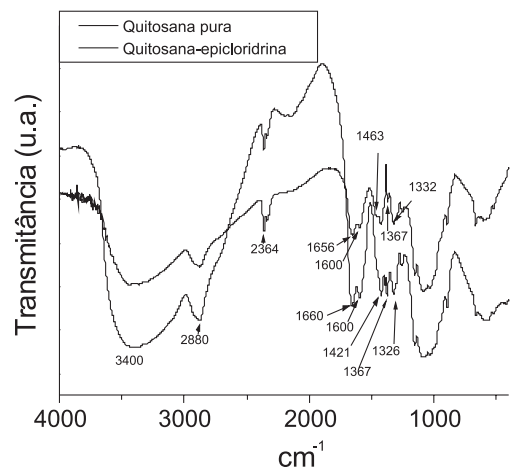

Figura 2. Espectros de FTIR das esferas de quitosana antes e após reticulação com epicloridrina

As diferenças mais significativas entre os dois espectros ocorreram na faixa de $1000-2000 \mathrm{~cm}^{-1}$, onde são encontrados, principalmente, estiramentos do tipo $\mathrm{C}-\mathrm{O}$, os quais resultaram da reação da quitosana com a epicloridrina. Entre $1300-1400 \mathrm{~cm}^{-1}$ encontram-se deformações do tipo C-H. A pequena banda em $1463 \mathrm{~cm}^{-1}$ no espectro das esferas reticuladas é atribuída ao estiramento de amina secundária N-H. Essa observação parece evidenciar que a reação da epicloridrina com as esferas também ocorreu, provavelmente em pequena extensão, utilizando alguns grupos amina da quitosana. ${ }^{13}$

\section{Estudos de adsorção de $\operatorname{Cr}(\mathrm{VI})$ nas esferas}

Os processos de adsorção em interfaces sólido/solução são relativamente complexos, uma vez que várias espécies podem adsorver sobre a superfície do adsorvente. Em estudos anteriores, determinouse que as interações de espécies aniônicas oriundas do íon cromato com quitosanas ocorrem, preferencialmente, por interações eletrostáticas utilizando-se sítios de adsorção carregados positivamente, os quais se originam dos grupos orgânicos oxigenados e nitrogenados presentes na quitosana. As maiores adsorções de cromatos na quitosana ocorrem na faixa ácida da escala de $\mathrm{pH}$, tipicamente entre 3,5 a

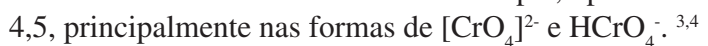

Os valores das quantidades adsorvidas de $\mathrm{Cr}(\mathrm{VI})$, bem como dos parâmetros cinéticos e termodinâmicos são mostrados na Tabela 1. Um exemplo típico do perfil das curvas calorimétricas obtidas é mostrado na Figura 3. Inicialmente, nota-se o aumento progressivo das energias de

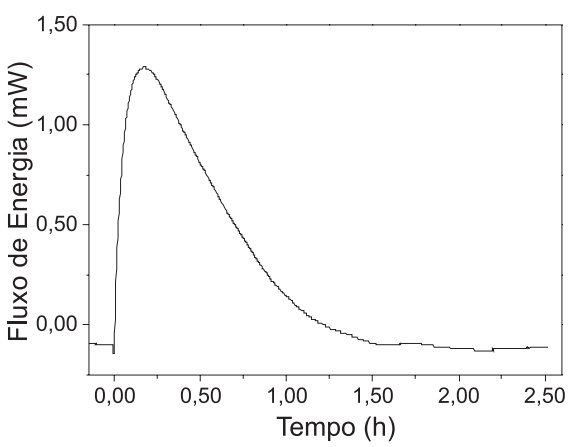

Figura 3. Exemplo de curva calorimétrica obtida nos processos de adsorção de Cr(VI) nas esferas de quitosana reticuladas em função do tempo de contato
Tabela 1. Parâmetros de adsorção de Cr(VI) nas esferas de quitosana. Resultados mostrados em duplicata

\begin{tabular}{lccccc}
\hline $\begin{array}{l}\mathrm{C}_{\mathrm{i}} \text { de Cr(VI) } \\
\left(\mathrm{mol} \mathrm{dm}^{-3}\right)\end{array}$ & $\begin{array}{c}\mathrm{T} \\
(\mathrm{K})\end{array}$ & $\begin{array}{c}\mathrm{N} / 10^{-5} \\
\left(\mathrm{~mol} \mathrm{~g}^{-1}\right)\end{array}$ & $\begin{array}{c}-\mathrm{Q}_{\mathrm{r}} \\
\left(\mathrm{J} \mathrm{g}^{-1)}\right.\end{array}$ & $\begin{array}{c}\text { Tempo } \\
\text { de reação } \\
(\mathrm{min})\end{array}$ & $\begin{array}{c}-\Delta_{\text {ads }} \mathrm{H} \\
\left(\mathrm{kJ} \mathrm{mol}^{-1}\right)\end{array}$ \\
\hline $5,0 \times 10^{-3}$ & 308 & 6,71 & 2,00 & 150 & 29,84 \\
& 308 & 6,96 & 2,31 & 145 & 33,22 \\
& 318 & 7,35 & 1,72 & 97 & 23,43 \\
$5,0 \times 10^{-4}$ & 318 & 7,13 & 1,90 & 96 & 26,75 \\
& 308 & 0,56 & 0,80 & 118 & 142,58 \\
& 308 & 0,78 & 0,90 & 117 & 117,22 \\
& 318 & 0,29 & 1,03 & 80 & 363,86 \\
& 318 & 0,23 & 1,04 & 85 & 453,98 \\
\hline
\end{tabular}

Estimativa do desvio padrão médio de $3 \%$ para os resultados calorimétricos diretos $\left(\mathrm{Q}_{\mathrm{r}}\right.$ e tempo de reação e de 5,7\% para os resultados indiretos $(\mathrm{N}$ e $\Delta \mathrm{H})$.

adsorção relacionadas à ocupação dos sítios de adsorção mais energéticos das esferas de quitosana. Contudo, todas as curvas calorimétricas se apresentaram como gaussianas com assimetrias consideráveis, evidenciando heterogeneidades energéticas dos sítios de adsorção nas esferas de quitosana. ${ }^{14}$ Essas diferenças nas energias de interação dos sitos de adsorção são decorrentes da dependência existente entre os parâmetros de adsorção, ou seja, entre a quantidade adsorvida, a energia de adsorção e a fração de ocupação dos sítios de adsorção de um adsorvente sólido $(\theta)$. As oscilações observadas das linhas de base nos finais das curvas calorimétricas são características de processos de adsorção/dessorção, que ocorrem quando o equilíbrio químico é alcançado. ${ }^{19}$

A entalpia de adsorção $\left(\Delta_{a d s} H\right)$ relaciona os valores de $N$ e $Q_{i n}$ de um dado processo de adsorção, como mostrado na Equação 3..$^{15}$ Termodinamicamente, a relação de dependência entre $\Delta_{a d s} H$, a temperatura $(T)$ e $\theta$ (entre 0 e 1) é mostrada na Equação 4:20

$\Delta_{\text {ads }} H=\frac{Q_{\text {int }}}{N}$

$-\Delta_{a d s} H(T, \theta)=\int_{0}^{1} Q_{t}(T, \theta) d \theta$

Examinando-se os dados da Tabela 1, observa-se uma diminuição dos valores de $\Delta_{\text {ads }} H$ com o aumento de $N$ e, consequentemente, também com o aumento progressivo de $\theta$. Tipicamente, para processos de adsorção na interface sólido/solução, os valores de $N$ aumentam de forma mais significativa em relação aos valores de $Q_{i n t}$, à medida que a concentração inicial do adsorvato na solução aumenta, pois as energias dos sítios de adsorção em adsorventes sólidos não mudam de maneira significativa com o aumento de $\theta .{ }^{15}$

Nota-se que as variações nas concentrações iniciais de $\mathrm{Cr}(\mathrm{VI}) \mathrm{e}$ nas temperaturas de adsorção alteraram significativamente as quantidades adsorvidas de $\mathrm{Cr}(\mathrm{VI})$, bem como os parâmetros cinéticos e termodinâmicos calculados. As maiores quantidades de $\mathrm{Cr}(\mathrm{VI})$ adsorvido ocorreram nos experimentos realizados com as maiores concentrações iniciais do metal em solução. Isso pode ser explicado levando-se em consideração os processos de equilíbrio que ocorrem na interface sólido/solução, o qual é mostrado, de maneira genérica, na Equação 5: ${ }^{13}$

quitosana (solv) $+\mathrm{Cr}(\mathrm{VI}) \rightleftharpoons$ quitosana $-\mathrm{Cr}(\mathrm{VI})($ solv $)+$ solv

Sabe-se que vários processos químicos ocorrem simultaneamente, até que o equilíbrio adsorvente/adsorvato seja atingido, principalmente aqueles relacionados à remoção de parte das espécies químicas que 
solvatam o adsorvato e os sítios de adsorção do adsorvente. Sempre quando ocorre aumento na concentração do adsorvato em solução, o equilíbrio estabelecido é deslocado para a direita, no sentido de aumentar a quantidade adsorvida pelo adsorvente. ${ }^{13,15}$

$\mathrm{Na}$ Tabela 1 observa-se, ainda, que os tempos dos experimentos calorimétricos de adsorção variaram entre 80 e 150 min. A temperatura de adsorção parece apresentar influência menos significativa nos parâmetros de adsorção $N, Q_{i n t}$ e $\Delta_{a d s} H$. Porém, se nota que os maiores tempos de reação ocorreram nos experimentos realizados na menor temperatura, $308 \mathrm{~K}$. Portanto, os processos de adsorção, em relação ao tipo de resposta observada, apresentam comportamentos cujas análises são relativamente complexas, onde as variáveis concentração inicial de $\mathrm{Cr}(\mathrm{VI})$ em solução e a temperatura podem atuar de maneira sinérgica ou antagônica entre si, dependendo do tipo de resposta avaliada para os processos de adsorção.

$\mathrm{Na}$ tentativa de avaliar os resultados obtidos de maneira mais clara, foi realizada uma análise multivariada dos resultados calorimétricos, utilizando-se um planejamento fatorial $2^{2}$, para analisar os efeitos das variáveis concentração inicial de $\mathrm{Cr}(\mathrm{VI})$ em solução $\left(C_{i}\right)$ e a temperatura de adsorção $(T)$, e de suas interações binárias. ${ }^{21}$ As respostas dos planejamentos foram os parâmetros relacionados às quantidades adsorvidas de $\mathrm{Cr}(\mathrm{VI})(N)$, aos tempos dos processos $\left(T_{r}\right)$ e às variáveis termodinâmicas $Q_{i n t}$ e $\Delta_{a d s} H$. Os resultados obtidos são mostrados na Tabela 2. A partir da análise dos valores dos efeitos calculados e de seus respectivos erros, observa-se que os parâmetros $N$ e $Q_{r}$ são fortemente influenciados pelo efeito principal de $C_{i}$ e pela interação binária entre $C_{i}$ e $T$. A temperatura não mostrou efeito significativo para esses parâmetros. No entanto, os efeitos principais da $C_{i}$ e $T$, bem como das suas interações binárias, são todos estatisticamente importantes nos processos de adsorção de $\mathrm{Cr}(\mathrm{VI})$ nas esferas, em relação aos parâmetros $T_{r}$ e $\Delta_{a d s} H$. Ressalta-se que as correlações cinéticas e termodinâmicas observadas somente puderam ser obtidas e discutidas pelo uso da técnica da microcalorimetria isotérmica contínua.

Tabela 2. Resultados da análise multivariada da adsorção de $\mathrm{Cr}(\mathrm{VI})$ nas esferas de quitosana reticuladas

\begin{tabular}{|c|c|c|c|c|c|c|}
\hline \multirow{2}{*}{ 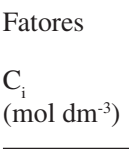 } & \multirow[b]{2}{*}{$\begin{array}{c}\mathrm{T} \\
(\mathrm{K})\end{array}$} & \multicolumn{5}{|c|}{ Efeitos observados \pm erros dos efeitos } \\
\hline & & $\begin{array}{c}\text { Tipo do } \\
\text { efeito }\end{array}$ & $\begin{array}{c}\mathrm{N} / 10^{-5} \\
\left(\mathrm{~mol} \mathrm{~g}^{-1}\right)\end{array}$ & $\begin{array}{c}-\mathrm{Q}_{\mathrm{r}} \\
\left(\mathrm{J} \mathrm{g}^{-1}\right)\end{array}$ & $\begin{array}{l}\text { Tempo de } \\
\text { reação } \\
(\min )\end{array}$ & $\begin{array}{c}-\Delta_{\mathrm{ads}} \mathrm{H} \\
\left(\mathrm{kJ} \mathrm{mol}^{-1}\right)\end{array}$ \\
\hline $5,0 \times 10^{-4}$ & 308 & Média & $\begin{array}{c}3,75 \\
\pm 0,05\end{array}$ & $\begin{array}{r}1,46 \\
\pm 0,05\end{array}$ & $\begin{array}{c}111 \\
\pm 0,90\end{array}$ & $\begin{array}{r}148,86 \\
\pm 11,74\end{array}$ \\
\hline $5,0 \times 10^{-3}$ & 308 & $\mathrm{C}_{\mathrm{i}}$ & $\begin{array}{c}6,57 \\
\pm 0,10\end{array}$ & $\begin{array}{c}1,04 \\
\pm 0,09\end{array}$ & $\begin{array}{c}22 \\
\pm 1,8\end{array}$ & $\begin{array}{r}241,10 \\
\pm 23,44\end{array}$ \\
\hline $5,0 \times 10^{-4}$ & 318 & $\mathrm{~T}$ & $\begin{array}{l}-0,002 \\
\pm 0,10\end{array}$ & $\begin{array}{c}-0,08 \\
\pm 0,09\end{array}$ & $\begin{array}{l}-43 \\
\pm 1,8\end{array}$ & $\begin{array}{l}-136,29 \\
\pm 23,44\end{array}$ \\
\hline $5,0 \times 10^{-3}$ & 318 & $\mathrm{C}_{\mathrm{i}} \times \mathrm{T}$ & $\begin{array}{c}0,41 \\
\pm 0,10\end{array}$ & $\begin{array}{c}-0,26 \\
\pm 0,09\end{array}$ & $\begin{array}{c}-8,0 \\
\pm 1,8\end{array}$ & $\begin{array}{r}142,73 \\
\pm 23,44\end{array}$ \\
\hline
\end{tabular}

Foram realizados alguns testes de modelagem cinética de adsorção, utilizando-se a isoterma cinética resultante da somatória dos fluxos de calor das curvas calorimétricas obtidas, conforme pode ser observado na Figura 4. Os dados da isoterma cinética foram ajustados a um modelo matemático exponencial, o qual contempla o cálculo das ordens e das constantes cinéticas de velocidade, utilizando-se a Equação 6:17

$Q_{\text {int }}=Q_{e} \cdot\left(1-\exp ^{\left[-[k . t]^{n}\right.}\right)$

onde $Q_{e}$ é a quantidade máxima do fluxo de energia registrado dos processos de adsorção, $k$ é a constante de velocidade $\left(\min ^{-1}\right)$ e $n$ representa a ordem dos processos cinéticos de adsorção. De modo geral, o modelo exponencial apresentou bons ajustes $\left(\mathrm{R}^{2} \geq 0,998 \mathrm{e}\right.$ Qui-quadrado $<10^{-10}$ ), com valores médios de $n$ e $k$ de 1,65 e 4,50 x $10^{-4} \mathrm{~min}^{-1}$, respectivamente.

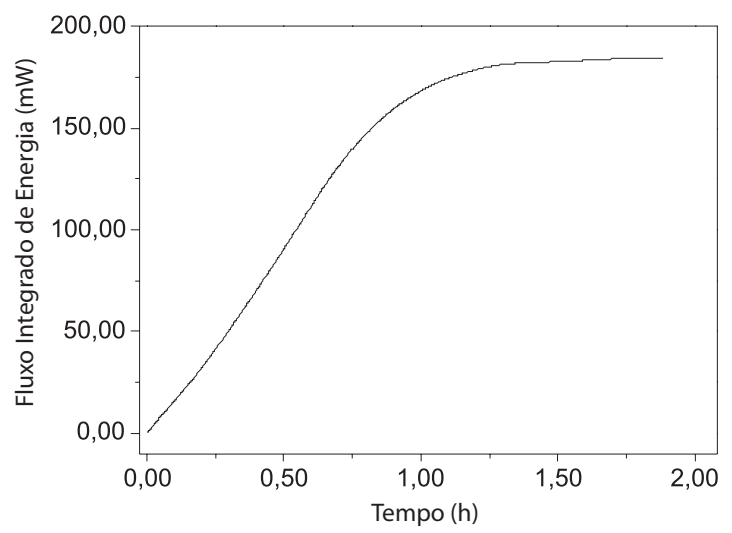

Figura 4. Exemplo de isoterma cinética obtida a partir das curvas calorimétricas dos processos de adsorção de $\mathrm{Cr}(\mathrm{VI})$ nas esferas de quitosana reticuladas

\section{CONCLUSÃO}

Neste trabalho, esferas de quitosana foram sintetizadas e caracterizadas, evidenciando-se diferenças químicas e estruturais significativas, antes e após reticulação com epicloridrina. Avaliaram-se processos de adsorção de $\mathrm{Cr}(\mathrm{VI})$ nas esferas reticuladas, utilizando-se microcalorimetria isotérmica contínua. Essa técnica possibilitou o cálculo e análise de novas correlações entre parâmetros cinéticos e termodinâmicos de adsorção que ocorrem nas interfaces sólido/solução. Todos os processos de adsorção foram exotérmicos. A análise estatística multivariada evidenciou a existência de interações binárias importantes entre as variáveis, afetando significativamente os valores numéricos dos tempos dos processos cinéticos e das entalpias de adsorção $\left({ }_{a d s} H\right)$. A modelagem cinética mostrou que os processos cinéticos de adsorção ocorrem, tipicamente, com ordens cinéticas fracionárias.

\section{AGRADECIMENTOS}

M. A. S. Andrade Jr agradece ao CNPq pelas bolsas (IC/PIBIC). A. R. Cestari agradece ao $\mathrm{CNPq}$ pela bolsa de Produtividade em Pesquisa e pelo apoio financeiro e à CAPES pelo apoio financeiro e pela bolsa de Pós-Doutorado.

\section{REFERÊNCIAS}

1. Muzzarelli, R. A. A.; Carbohydr. Polym. 2009, 76, 167.

2. Kasaai, M. R.; J. Agric. Food Chem. 2009, 57, 1667.

3. Guibal, E.; Sep. Purif. Technol. 2004, 38, 43.

4. Guibal, E.; Prog. Polym. Sci. 2005, 30, 71.

5. Curti, E.; Campana-Filho, S. P.; J. Macromol. Sci. Pure Appl. Chem. 2006, $A 43,555$.

6. Sinha, V. R.; Singla, A. K.; Wadhawan, S.; Kaushik, R.; Kumria, R.; Bansal, K.; Dhawan, S.; Int. J. Pharm. 2004, 274, 1.

7. Miao, J.; Chen, G.; Gao, C.; Dong, S.; Desalination 2008, 233, 147.

8. Chatterjee, S.; Lee, D. S.; Lee, M. W.; Woo, S. H.; Bioresour. Technol. 2009, 100, 2803.

9. Ganjali, M. R.; Babaei, L. H.; Badiei, A.; Saberian, K.; Behbahani, S.; Ziarani, G. M.; Niasari, M. S.; Quim. Nova 2006, 29, 440.

10. Andrade, P. S.; Lima, C. A. P.; Sousa, J. T.; Lima, G. G. C.; Vieira, F. F.; Quim. Nova 2007, 5, 1082.

11. Deepika, S.; Buddhi, D.; Int. J. Environ. Pollut. 2006, 27, 324.

12. Rao, R. A. K.; Khan, M. A.; Colloids Surf. A 2009, 332, 121. 
13. Cestari, A. R.; Vieira, E. F. S.; Mota, J. A.; J Hazard. Mater. 2008, 160, 337.

14. Cestari, A. R.; Vieira, E. F. S.; da Rocha, F. C.; Thermochim. Acta 2005, 430, 211.

15. Vieira, E. F. S.; Cestari, A. R.; Airoldi, C.; Loh, W.; Biomacromolecules 2008, 9, 1195.

16. Boni, M. R.; Sbaffoni, S.; J. Hazard. Mater. 2009, 166, 1087.

17. Cestari, A. R.; Vieira, E. F. S.; Tavares, A. M. G.; Bruns, R. E.; J. Hazard. Mater. 2008, 153, 566.
18. Pillai, C. K. S.; Paul, W.; Sharma, C. P.; Prog. Polym. Sci. 2009, 34, 641.

19. Vieira, E. F. S.; Cestari, A. R.; da Silva, R. G.; Pinto, A. A.; Miranda, C. R.; Conceição, A. C. F.; Thermochim. Acta 2004, 419, 45.

20. Garcia-Cuello, V.; Moreno-Piraján, J. C.; Giraldo-Gutiérrez, L.; Sapag, K.; Zgrablich, G.; Microporous Mesoporous Mater. 2009, 120, 239.

21. Barros Neto, B.; Scarmínio, I. S.; Bruns, R. E.; Como fazer experimentos, Pesquisa e desenvolvimento na ciência e na indústria, $2^{\mathrm{a}}$ ed., Ed. da Unicamp: Campinas, 2001 